Well that changes everything! The genesis of memory bias for threat with implications for delayed onset in anxiety disorders

Jessica M. Senn a and Adam S. Radomsky*a

${ }^{\mathrm{a} C o n c o r d i a}$ University

Department of Psychology

7141 Sherbrooke St. W.

Montreal, QC, Canada, H4B 1R6

* Corresponding Author. Tel.: +1 514848 2424/2202; fax: +1 5148484523. E-mail address: adam.radomsky@concordia.ca (A.S. Radomsky) 


\begin{abstract}
Background and Objectives: A number of aetiological pathways have been proposed in the development of anxiety disorders, including those associated with stressful triggering situations. Life events can provide new meaning to past situations, potentially leading to the delayed onset of a disorder. Whether or not a disorder will emerge is theoretically related to one's appraisal and memory of prior events, and memory biases are proposed to exist for threat-related information in association with anxiety. Given that new events may change the meaning of past experiences, threatening information may change one's memory for once-neutral events.

Methods: The current study aimed to examine the effect of threatening information on memory for previously encoded (neutral) stimuli. Undergraduate participants $(n=81)$ interacted with 30 neutral objects (displayed in two boxes) and completed a recall memory test for these objects. They were then randomly assigned to receive either new threatening or new neutral information about half (one box) of the already-learned objects; a second recall test was then administered. Results: Individuals given threatening information showed a greater proportion of memory for items that were manipulated to items that remained neutral than did individuals given new nonthreatening information. Limitations: A nonclinical sample reported relatively low ratings of disgust and anxiety. Additionally, the time between the two memory tests was brief, likely differing from the actual occurrence of delayed onset disorders. Conclusions: Results showed the genesis of a memory bias for threat in the presumed absence of an attentional bias, and are discussed in terms of the delayed onset of anxiety disorders.
\end{abstract}

KEYWORDS: Memory bias; anxiety disorders; delayed onset; threat; information processing. 


\section{Well that changes everything! The genesis of memory bias for threat with implications for delayed onset in anxiety disorders}

Anxiety disorders are thought to have a number of different aetiological pathways, including those associated with stressful triggering situations (Rachman, 1977). Theories implicating fear conditioning in the genesis of anxiety disorders have been studied for decades

(for a review, see Lissek et al., 2005), and suggest that the development of anxiety disorders can be related to the classical conditioning of a fear response, often based on the occurrence of specific triggering events. Alternate pathways include vicarious conditioning, informational acquisition, or fears that are 'prepared' (Rachman, 1977). In many individuals, a onceconditioned fear will be extinguished, but in others fear becomes their primary response in situations involving the conditioned stimulus, and pathological anxiety can result (Eysenck, 1979). In typical cases of classical and vicarious conditioning, fear is conditioned during or immediately following the occurrence of the conditioning event(s). Surprisingly, psychological disorders can develop well after an individual encounters a specific stressor or situation that one would normally construe as potentially responsible for the onset of the problem. For example, in a small subset of cases of posttraumatic stress disorder (PTSD), the disorder does not develop until after months, or even years have passed (PTSD With Delayed Onset; DSM-IV 309.81; APA, 1994, p.465). Although the mechanisms involved in some aetiological pathways of the development of anxiety disorders are somewhat understood, it is important to gain a better understanding of alternate mechanisms involved in the development of anxious psychopathology.

The majority of evidence for the occurrence of disorders with delayed onset stems from research in the area of PTSD, presumably due to the ease of identifying the triggering event. 
However, there are anecdotal reports in which individuals develop non-PTSD fear-related problems after a substantial amount of time has passed since the triggering event. For example, one client reported an extreme fear of bee stings which began at least eight years following her only experience of a bee sting (during which she denied the presence of fear or avoidance), and emerged only after having viewed a documentary about the production of honey. This anecdotal account provides support for the suggestion that fears can emerge suddenly with no apparent (recent) traumatic experience (Marks, 1969; Rachman, 1977). Although it has been established that delayed onset occurs in PTSD, based on the above example as well as a number of other similar anecdotal descriptions, it is expected that other disorders can also have a delayed onset. Of course, it cannot be assumed that delayed onset in PTSD is comparable to the occurrence of delayed onset following a less traumatic, yet negative event; however, it is likely that mechanisms leading to delayed onset after different types of events would be similar.

It has been proposed that previous traumas may gain a new, more threatening meaning due to experiences following the traumatic event, which may account for the delayed onset of PTSD in individuals who initially did not meet criteria for the disorder (Ehlers \& Clark, 2000; Ehlers, Mayou, \& Bryant, 1998; Herrmann \& Eryavec, 1994; Soloman et al., 1989). This reappraisal of threat may be an important factor in delayed onset PTSD. It remains to be seen whether or not threat (re)appraisal may relate to delayed onset in other psychological disorders.

Memory for events in one's life, especially for those events which were interpreted as threatening, is an important factor in the development and maintenance of PTSD (Amir, Leiner, \& Bomyea, 2010; Robinaugh \& McNally, 2010) and of many other anxiety disorders such as social phobia (e.g., Cody \& Teachman, 2010; Morgan, 2010), obsessive-compulsive disorder (OCD; e.g., Radomsky \& Rachman, 2004) and panic disorder (e.g., Hagenaars, van Minnen, 
Hoogduin, \& Verbraak, 2009). Whether or not individuals will develop these disorders relates to both their memory and appraisal(s) of these events (i.e., what meaning they give to the events; Ehlers \& Clark, 2000). Given that Ehlers and Clark have suggested that additional events occurring after an initial trauma can change the meaning of that trauma and potentially cause the onset of PTSD, it is possible that non-traumatic, but still threatening information can impact or change one's memory for what were once neutral events.

Memory biases associated with anxious arousal likely have an effect on one's memory for threat-related situations. Cognitive psychology theories predict the presence of memory biases within the context of emotional arousal (e.g., Bower, 1981), such as low mood and anxiety. According to Bower's theory, emotionally arousing information will result in an increased allocation of attentional and memorial resources to this information. Presumably, this understanding further extends to the experience of other negative reactions such as disgust, especially since disgust has been shown to be related to anxiety in a variety of anxiety disorders (for a review, see Olatunji, Cisler, \& Phillips, 2010).

A number of studies have demonstrated a memory bias for threat-related information. For example, Radomsky and Rachman (1999) compared a sample of contamination fearful participants diagnosed with OCD to anxious and nonanxious controls and found that they had greater memory for contaminated (versus noncontaminated) objects, whereas the control groups did not. There have been additional studies showing memory biases in OCD (e.g., Radomsky, Rachman, \& Hammond, 2001) as well as other anxiety-related problems such as panic disorder (e.g., Cloitre \& Liebowitz, 1991), social phobia (e.g., Ashbaugh \& Radomsky, 2009, 2011) and generalized anxiety disorder (e.g., Coles, Turk, \& Heimberg, 2007). In addition to these studies which have empirically demonstrated a memory bias for anxiety-provoking stimuli, anxious 
patients often report powerful and highly detailed memories of specific events in which they were extremely anxious/fearful.

The current study examined memory processing and cognitive change. Since cognitive change related to anxiety and fear is common in cases of delayed onset PTSD, this study was designed to test whether or not this is also present in association with other types of anxiety. Specifically, the current study examined whether or not memory for neutral stimuli could be altered by the addition of new information that made once-neutral stimuli become threatening. Demonstrating this empirically would provide much-needed information about one of many possible mechanisms involved in the aetiology of anxiety disorders - especially in the genesis of anxiety disorders with delayed onset. This would also provide important information about fear generalization, and a pathway to disorder acquisition that has yet to be studied outside of PTSD. In the current study, we employed a delayed tarantula as the threatening stimulus. The tarantula acted as a stimulus that added threat value to initially neutral objects (to parallel how events in one's life may add threat to a previously neutral situation), and changes in memory for the onceneutral objects were assessed. Thus, it was an experimental investigation of delayed onset in anxiety unrelated to PTSD.

We hypothesized that if neutral stimuli were encoded and then made threatening at a later time (through the provision of new threatening information), there would be a subsequent proportionate increase in memory (i.e., memory bias) for stimuli that became threatening compared to those that remained neutral. We also hypothesized that the provision of new neutral information would not result in greater memory for any items. Given that memorial systems have a limited capacity (Miller, 1956) that is unlikely to be surpassed regardless of the threat level of the new information, it was expected that overall recall memory performance would not differ 
between conditions, but that the proportion of manipulated items remembered (to total number of items remembered) would be higher in the threat condition than in the neutral condition. An increase in proportion of memory for threat-related stimuli would indicate a change in memory due to the introduction of threat, and represent a memory bias for threat-related information. It would also presumably occur in the absence of attentional bias, since all stimuli were neutral throughout encoding. Given that novel strategies are being developed for the treatment of anxiety disorders via the manipulation/training of attentional biases, further information about the role of memory biases in anxiety disorders, and especially those with delayed onset, would suggest that novel treatment strategies based on memory may warrant development and testing.

\section{Method}

\section{Participants}

A total of 120 undergraduate students participated in the study and were compensated with course credit; however, data from five of these individuals were not included in the analyses due to either very poor memory performance $(n=1)$, or extreme levels of distress during the manipulation phase of the study $(n=4)$, as determined using standard outlier exclusion methods (Tabachnick \& Fidell, 2007). Additionally, participants who indicated that they did not believe the manipulation at all $(n=34)$ were removed from the analyses. Data from the remaining 81 participants were retained. Participants ranged in age from 18 to $57(M=24.00, S D=7.15)$ years. The majority of participants were female ( $88 \%)$ and identified themselves as Caucasian (75\%). Participants were randomly assigned to either a Threat or No-Threat condition (see Procedures below). Following this, there were 33 individuals in the Threat condition, with a mean age of $25.18(S D=8.72)$ years. Eighty-two percent of the individuals in the Threat condition were female. The No-Threat condition consisted of 48 individuals with a mean age of 
$23.19(S D=5.79)$ years, 92 percent of whom were female. There were no significant differences between the two conditions in terms of age, $t(113)=1.15, p=.26$, or sex, $\chi^{2}(1)=1.75, p=.19$.

\section{Measures}

Recall memory test. On two occasions during the study participants completed a free recall memory test. They were given three minutes to verbally name as many objects as they could remember from the objects presented in the two boxes earlier in the study (see Procedures below). The experimenter recorded their answers verbatim.

Current Emotional State. Rating scales parallel to the Subjective Units of Distress Scale (SUDS; Wolpe, 1958) were used to assess the current emotional state of participants. Specifically, participants were asked to use a 0-100 scale to rate their current negative emotions such as level of anxiety (typical SUDS rating), urge to wash their hands, and disgust (feelings of disgust as well as how willing they would be to eat their lunch out of each of two boxes shown to them earlier). Participants were also asked about other mood states (e.g., happiness, fatigue) in order to draw focus away from questions pertaining to anxiety and disgust and to disguise the purpose of the study, and were therefore intended to increase believability of the manipulation.

Vancouver Obsessional Compulsive Inventory (VOCI; Thordarson et al., 2004). The VOCI is a 55-item scale that assesses a range of obsessive compulsive symptoms such as checking, contamination, obsessions, etc. Test-retest reliability in a student sample was shown to be 0.91 , and the internal consistency for the VOCI is $\alpha=0.96$ (Radomsky et al., 2006). The VOCI also has good convergent/divergent validity. The internal consistency for the VOCI in the current study was $\alpha=0.94$.

Disgust Scale (DS; Haidt, McCauley, \& Rozin, 1994). The DS is a 32-item scale measuring an individual's sensitivity to a variety of disgust-related stimuli. Inter-item reliability 
has been shown to be excellent (Cronbach's $\alpha=0.84$ ). The internal consistency for the DS in the current study was $\alpha=0.76$.

Fear of Spiders Questionnaire (FSQ; Szymanski \& O’Donohue, 1995). The FSQ is an 18-item questionnaire designed to assess spider-related fears. The internal consistency for the FSQ is $\alpha=0.92$. Additionally, this scale has been demonstrated to be useful when assessing low levels of fear, and is therefore a good questionnaire to use when studying nonclinical samples (Muris \& Merckelbach, 1996). The internal consistency for the FSQ in the current study was $\alpha=$ 0.97 .

Spider Phobia Beliefs Questionnaire (SBQ; Arntz, Lavy, van den Berg, \& van Rijsoort, 1993). The SBQ is a 78-item scale that assesses fearful beliefs about spiders as well as one's reaction to encountering spiders. The scale is composed of two subscales: the spider-related beliefs subscale, and the self-related beliefs subscale. Both subscales have demonstrated good internal consistency, both with Cronbach's $\alpha$ 's $=0.94$. The internal consistency for the SBQ in the current study was $\alpha=0.98$.

Beck Depression Inventory-2 (BDI-II; Beck, Steer, \& Brown, 1996) and Beck Anxiety Inventory (BAI; Beck \& Steer, 1990). The BDI-II and BAI are well used 21-item questionnaires assessing symptoms of depression and anxiety respectively. The internal consistencies of these scales in the current study were $\alpha$ 's $=0.89$ and 0.88 , respectively.

Manipulation Believability Questionnaire (MBQ). The MBQ is an 8-item questionnaire created for this study based on similar measures previously used by our team. It assessed the believability of the manipulation (e.g., "Based on the information you were given, how convinced are you that the [paper/spider] is usually kept in one of the boxes?"), as well as how distressed they were by the manipulation (e.g., "How upset were you about the 
[paper/spider] having been in one of the boxes of objects you touched?"). Participants responded to each question by selecting one of five multiple choice responses (e.g., definitely, mostly, somewhat, a little, or not at all convinced). Each condition had its own version, differing only in the wording of questions specifically addressing the manipulation (i.e., spider versus paper).

\section{Materials}

The stimuli used in this study were 30 small objects purchased from a dollar store (see Appendix). Each object fit the following criteria: no larger than $10 \mathrm{~cm}$ in its largest dimension, at least one dimension larger than $4 \mathrm{~cm}$, easily nameable (i.e., identifiable by the general population), not clearly associated with contamination (e.g., a sponge), and not too similar to other objects in the study (i.e., not easily confused with other study objects).

Two cardboard boxes with lids were used in the study. They were $30.5 \mathrm{~cm}$ by $38.1 \mathrm{~cm}$ by $25.4 \mathrm{~cm}$ in size, and were identical other than being different colours (brown and white). The paper presented to the control group was a stack of three packages of plain white printer paper, still in their original wrapping. The tarantula presented in the threat condition was a live Chilean Rose tarantula, presented in a clear terrarium containing soil and two water dishes in the corners.

\section{Procedure}

Participants were informed that they were taking part in a study aiming to expand upon previous research on free association. The task they completed consisted of picking up a series of 30 objects from two separate boxes (one white, one brown; 15 items per box). The participants were asked to pick up the objects one at a time, alternating between the two boxes, with the experimenter dictating the order in which the objects were to be picked up. They were further instructed to use the hand that corresponded to box location (e.g., use their left hand for objects in the box on their left) when picking up the objects. 
The following variables were counterbalanced across all participants: side of the table on which each box was presented, group of items in each box, order in which each set of objects was presented, side that participants were asked to take the first item from, and which box (left or right) would become the manipulated box. This was done in order to ensure that effects found in this study could not be readily attributed to order or location effects.

For each object they picked up, participants were asked to generate and say out loud a novel sentence describing the object, and then place the item back in its original box. These sentences were recorded verbatim by the experimenter. After the free association task, participants completed a distractor task in which they were asked to count backwards out loud in sevens for 3.5 minutes, starting at 46,305. Following the distractor task, a baseline recall memory test was administered.

Following the baseline recall test, participants were given a break and were then provided with additional information about the two boxes. All participants were told that one of the boxes is only used for the purposes of this study, which will be referred to as the un-manipulated box from this point forward. Participants were randomly assigned to either the experimental (Threat) condition, or the control (No-Threat) condition. The experimenter was blind to group assignment until this point in the study. Condition assignment determined what participants were told were the typical contents of the second box, which will be referred to as the manipulated box from this point forward. In the No-Threat condition, the experimenter brought a stack of packaged paper into the testing room and told the participant that the paper was typically kept in the box and had to be removed each time the study was conducted. In the Threat condition, the experimenter brought the tarantula into the room and told the participant that the spider 'plays' in the box when we clean his tank. A detailed script was followed for the provision of the new information 
in each condition ${ }^{1}$. Following the manipulation, participants were asked to provide information about their current emotional state, and then completed a second recall memory test.

Participants concluded the study by filling out a number of self-report questionnaires (see Measures above). Participants were then debriefed about the true purpose of the study as well as the rationale for the use of deception. Individuals in the experimental condition were informed that the tarantula never in fact touched the objects in the box and, for a small number of participants who were reportedly anxious due to the presence of the tarantula, were asked to remain in the laboratory until these feelings of anxiety had diminished. Additionally, all participants signed a second consent form agreeing that their data could be retained for use, now that they knew the real purpose of the study.

\section{Data analyses}

For main analyses of memory performance, the dependent variable of interest was calculated by dividing the number of items recalled from the manipulated box by the total number of items recalled. This value was calculated and entered for each participant at each time point prior to analysis. This proportionate memory variable was the chosen variable for analysis because it appeared to be an appropriate measure of memory performance over time while taking into account memory's limited capacity (Miller, 1956).

\section{Results}

\section{Participants}

Means and standard deviations for all self-report measures are reported in Table 1. There were no significant differences between the conditions on spider-related fears, obsessivecompulsive symptomatology, anxiety, or depressive symptomatology. A significant difference was found between conditions in terms of disgust sensitivity (Disgust Scale scores; $t(79)=-2.17$,

\footnotetext{
${ }^{1}$ The full script is available from the corresponding author.
} 
$p=.03$ ), with individuals in the No-Threat condition reporting a higher level of disgust sensitivity than those in the Threat condition. Due to this unexpected difference, scores on the Disgust Scale were entered as a covariate for relevant analyses.

\section{Manipulation check}

After the manipulation phase of the experiment, participants responded to a number of questions about their current feelings of anxiety, disgust, and contamination. As expected, there was a significant difference between conditions on both explicit $(t(79)=2.94, p=.006, d=$ 0.71), and less explicitly stated feelings of disgust (i.e., one's willingness to eat their lunch out of the un-manipulated versus the manipulated box; $t(79)=3.45, p=.002, d=0.85^{2}$ ), with participants in the Threat condition showing higher levels of disgust. There were no differences between conditions in terms of anxiety level, $t(79)=0.62, p=.53, d=0.14$, or feelings of contamination, $t(79)=-0.71, p=.48, d=-0.16$.

On the MBQ, individuals in the No-Threat condition reported being less upset by the regular use of the boxes than those in the Threat condition, $t(79)=-4.22, p<.001, d=-1.03$. Along with this expected difference between conditions, individuals in the Threat condition were less convinced by the information they were given, $t(79)=3.51, p=.001, d=0.78$. Although believability was lower in the Threat condition than in the No-Threat condition, individuals in the Threat condition on average reported being 'somewhat convinced' by the manipulation information. Therefore, these results suggest that the manipulation was at least partially believed by participants. As mentioned previously, individuals who reported not believing the manipulation at all (in either condition) were removed from the analyses.

\section{Memory Performance}

\footnotetext{
${ }^{2}$ Levene's test of homogeneity of variance was significant $(p<.05)$ for these manipulation check variables; therefore, $t$ and $d f$ values reported assume unequal variances.
} 
The mean number of items recalled at each time point, in each box, and by condition (as well as change scores over time) is listed in Table 2 below, along with proportionate memory for each condition. The mean number of items recalled in the first memory test was $18.52(S D=$ 2.83), and in the second memory test, it was $20.16(S D=2.92)$. A dependent samples $t$-test showed a significant increase in memory over time, $t(80)=7.89, p<.001, d=0.88$. This difference was evident in both the Threat condition, $t(32)=5.12, p<.001, d=0.90$, and the NoThreat condition, $t(47)=5.98, p<.001, d=0.86$.

\section{Overall analyses}

A planned comparison was conducted in order to understand the nature of the interaction between box type, condition, and time. The independent variable in this comparison was condition (Threat versus No-Threat), and the dependent variable was the proportionate memory for manipulated items compared to total item recall at time 2. A one-way ANCOVA was conducted in order to include Disgust Scale scores as a covariate (see above), as well as a covariate of the proportion of the number of items remembered from the manipulated box to the number of items remembered from the un-manipulated box at time 1 (i.e., initial memory performance). Results showed a significant difference between conditions, $F(1,80)=4.89, p=$ .03 , partial $\eta^{2}=.06$, with individuals in the Threat condition showing a higher proportion of manipulated items remembered than individuals in the No-Threat condition (see Figure 1$)^{3}$. The same difference between conditions was not present at time $1, F(1,80)=0.59, p=.45$, partial $\eta^{2}$ $=.01$.

\footnotetext{
${ }^{3}$ When disgust scale scores were not entered as a covariate, results still showed a significant difference in proportionate memory between conditions at time $2, F(1,80)=5.30, p=.02$, partial $\eta^{2}=.06$. When disgust scale scores were retained as a covariate but time 1 proportionate memory was removed, results remained significant, $F(1$, $80)=4.21, p=.04$, partial $\eta^{2}=.05$. Finally, when both covariates (disgust scale scores and time 1 proportionate memory) were removed, results still remained significant, $t(79)=2.20, p=.03, d=0.49$.
} 
Dependent samples $t$-tests were also carried out to investigate differences in memory for items from each box within each group at both time points. At time one, neither the Threat nor No-Threat condition showed a difference between memory for items from the two boxes, $t(32)=$ $1.06, p=.30, d=0.18$, and $t(47)=0.27, p=.79, d=0.04$, respectively. However, at time two individuals in the Threat condition showed greater memory for items that were in the manipulated box than for those that were in the un-manipulated box, $t(32)=2.04, p=.05, d=$ 0.36; this difference was not seen in the No-Threat condition, $t(47)=-0.40, p=.69, d=-0.05$.

\section{Discussion}

This study aimed to investigate whether the provision of threatening information related to previously-learned neutral stimuli could promote a memory bias for those stimuli. It was predicted that providing threatening information about neutrally encoded stimuli would cause an increase in memory for the now-threatening stimuli. Rather than assessing memory change via analysis of total numbers of items recalled, condition differences were examined based on the proportion of manipulated items remembered versus total memory recall following the manipulation. Individuals in the Threat condition showed a significantly higher proportion of manipulated objects remembered compared to individuals in the No-Threat condition. Individuals in the Threat condition also remembered significantly more manipulated than unmanipulated items, while there was no difference in memory for manipulated versus unmanipulated items in the No-Threat condition. Furthermore, these between-condition differences were not observed prior to the provision of new information. This is evidence for a memory bias for threat (despite the fact that no threat was present at encoding).

Overall these results provide evidence that a change in memory for previously-learned neutral objects can occur following the provision of threatening information. This appears to 
provide support for the theory that subsequent events occurring after encoding of information may lead to threat reappraisal (Ehlers \& Clark, 2000), although such appraisals were not directly assessed in the current study. The results of this study provide theoretical support for the notion that new information one receives about a specific past event can change memory for that event, and result in fear being attributed to that past event. Prior research shows that memory is important to the development and maintenance of anxiety disorders (e.g., Radomsky \& Rachman, 2004; Cody \& Teachman, 2010; Morgan, 2010; Robinaugh \& McNally, 2010), which leads to one hypothesis about how disorders of delayed onset may develop.

Interestingly, the current experiment reveals a memory bias in the presumed absence of an attentional bias. Bower's (1981) theory of information processing predicts both attentional and memory biases for threat-related information, and much research has shown that an increase in attention towards specific stimuli enhances the likelihood that those stimuli will be remembered (e.g., Christianson, 1992; MacLeod \& Mathews, 2004; Bar-Haim et al., 2007). It is therefore possible that the occurrence of a memory bias for threat normally results from increased attention at encoding. However, in the current study, all information was neutral at encoding; thus, the later increase in memory for threat-related information cannot be attributed to increased attention to these stimuli. Therefore, the results of the current study provide evidence that memory bias for threat is not simply a by-product of biased attention at encoding.

The current study is the first, we believe, to investigate possible mechanisms of delayed onset in anxiety other than PTSD. Although it cannot be assumed that delayed onset related to a traumatic event would parallel delayed onset in other anxiety disorders (related to a negative, but non-traumatic event), it is likely that they would show similar patterns of development. Furthermore, given that anecdotal evidence supports the possibility of other disorders developing 
with delayed onset, this novel area of research can further our understanding of the genesis of anxiety disorders. Additionally, it is to our knowledge the first known attempt at experimentally assessing possible cognitive mechanisms associated with the delayed onset of psychopathology, including fear generalization.

Although the results of the current study are promising, they are not without limitation. First, we tested a non-clinical sample, so its generalization to individuals diagnosed with anxiety disorders is questionable. Effect sizes were not large, which may be in part due to low levels of anxiety or disgust reactions across participants in general. With the manipulation causing low levels (on average) of distress, elucidating strong effects would be difficult.

This study was also limited by its short-term nature: participants completed both the preand post-manipulation memory tests within ten minutes. Given that when delayed onset is seen in PTSD it typically has an onset of months or years after the original event (Bryant \& Harvey, 2002; Carty, O'Donnell, \& Creamer, 2006), it would be useful to examine to what extent these effects would be evident given a greater length of time between the event, the time at which additional threatening information is provided, and when memory is assessed.

Another limitation to this study was the absence of pre-manipulation measures of anxiety. Due to the nature of the study, requesting anxiety ratings prior to the manipulation would have potentially exposed the true nature of the study and reduced believability. It is therefore difficult to determine whether or not there was a difference between groups in terms of change in anxiety due to the manipulation. However, given the differences in self-reported disgust (disgust is correlated with anxiety (e.g., Olatunji, Cisler, \& Phillips, 2010), especially in the context of spider-related fears (Olatunji, Huijding, de Jong, \& Smits, 2011)), disgust reactions are presumed to be an appropriate proxy for anxiety. It would, however, be important to include a pre- 
manipulation measurement of anxiety in further studies of this nature in order to assess changes in anxiety over time. It would also to be useful to examine these effects in participants with different types of threat concerns, and to investigate other factors that may impact the results of the experiment (e.g., valence or distinctiveness of the manipulation).

The results of this study may have a number of clinical implications. Knowing that disorders can develop after time has passed since a triggering event may be useful in identifying individuals who are at risk of developing a disorder, or particular types of interpretations that could lead to the onset of pathology related to previously-neutral experiences; this could lead to the possible prevention of anxiety disorders. Additionally, having an increased understanding of the mechanisms involved in the onset of a subset of disorders could provide opportunities to target specific therapeutic techniques, such as the reappraisal of anxious memories. This may be an important focus in treatment for individuals with delayed onset disorders, given that the disorder may have come about at least partially due to threat re-appraisal.

\section{Conclusions}

The present study demonstrated that individuals' memory for previously encoded neutral stimuli can change when those stimuli later become threatening. Results are consistent with findings from previous memory research (e.g., showing a memory bias for threat) and offer new support for the study of anxiety disorders with delayed onset, as well as potential mechanisms involved in their genesis. Although this study could not address many important questions related to disorders of delayed onset, it supports a combination of theories positing that delayed onset is not exclusively characteristic of PTSD. Further replication and investigation is necessary, but the current study provides results that further our understanding of the onset and 
maintenance of anxiety, as well as creating potential for advances in the prevention and treatment of anxiety disorders. 


\section{Acknowledgements/Author Notes}

This research was supported by the first author's Canada Graduate Scholarship Master's funding from the Canadian Institute of Health Research (CIHR), and by operating funds from the Natural Sciences and Engineering Research Council of Canada (NSERC) awarded to the second author. We express our gratitude to Katie Gordon Green, Stefanie Lavoie, Jeff Renaud, and Janice LaGiorgia for their help with participant recruiting and data collection, and to the anonymous reviewer, who provided a suggestion about an optimal way to compute proportionate memory. We would also like to thank Michel Dugas, Roisin O’Connor, and Hannah Levy for their helpful comments on earlier drafts of this manuscript. 


\section{References}

Amir, N., Leiner, A. S., \& Bomyea, J. (2010). Implicit memory for posttraumatic stress symptoms. Cognitive Therapy and Research, 34, 49-58.

American Psychiatric Association (1994). Diagnostic and statistical manual of mental disorders ( $4^{\text {th }}$ ed.) Washington, DC: Author.

Arntz, A., Lavy, E., van den Berg, G., \& van Rijsoort, S. (1993). Negative beliefs of spider phobics: A psychometric evaluation of the Spider Phobia Beliefs Questionnaire. Advances in Behaviour Research and Therapy, 15, 257-277.

Ashbaugh, A. R., \& Radomsky, A. S. (2009). Interpretations of and memory for bodily sensations during public speaking. Journal of Behavior Therapy and Experimental Psychiatry, 40, 399-411.

Ashbaugh, A. R., \& Radomsky, A. S. (2011). Memory for physiological feedback in social anxiety disorder: The role of fear of bodily sensations. Cognitive Therapy and Research, $35,304-316$.

Bar-Haim, Y., Lamy, D., Pergamin, L., Bakermans-Kranenburg, M. J., \& van IJzendoorn, M. H. (2007). Threat-related attentional bias in anxious and nonanxious individuals: A metaanalytic study. Psychological Bulletin, 133, 1-24.

Beck, A.T., \& Steer, R.A. (1990). Beck anxiety inventory manual. Toronto: Psychological Corporation.

Beck, A. T., Steer, R. A., \& Brown, G. K. (1996). Manual for the Beck Depression Inventory-II. San Antonio, TX: Psychological Corporation.

Bower, G. H. (1981). Mood and memory. American Psychologist, 36,129-148.

Bryant, R. A., \& Harvey, A. G. (2002). Delayed-onset posttraumatic stress disorder: A 
prospective evaluation. Australian and New Zealand Journal of Psychiatry, 36, 205-209.

Carty, J., O’Donnell, M. L., \& Creamer, M. (2006). Delayed-onset PTSD: A prospective study of injury survivors. Journal of Affective Disorders, 90, 257-261.

Christianson, S. (1992) Remembering emotional events: Potential mechanisms. In S.

Christianson (Ed.), The handbook of emotion and memory: Research and theory.

Hillsdale, NJ, England: Lawrence Erlbaum Associates, Inc.

Cloitre, M., \& Liebowitz, M. R. (1991). Memory bias in panic disorder: An investigation of the cognitive avoidance hypothesis. Cognitive Therapy and Research, 15, 371-386.

Cody, M. W., \& Teachman, B. A. (2010). Post-event processing and memory bias for performance feedback in social anxiety. Journal of Anxiety Disorders, 24, 468-479.

Coles, M. E., Turk, C. L., \& Heimberg, R. G. (2007). Memory bias for threat in generalized anxiety disorder: The potential importance of stimulus relevance. Cognitive Behaviour Therapy, 36, 65-73.

Ehlers, A., \& Clark, D. M. (2000). A cognitive model of posttraumatic stress disorder. Behaviour Research and Therapy, 38, 319-345.

Ehlers, A., Mayou, R. A., \& Bryant, B. (1998). Psychological predictors of chronic posttraumatic stress disorder after motor vehicle accidents. Journal of Abnormal Psychology, 107, 508519.

Eysenck, H. J. (1979). The conditioning model of neurosis. Behavioral and Brain Sciences, 2, 155-199.

Hagenaars, M. A., van Minnen, A., Hoogduin, C. A. L., \& Verbraak, M. (2009). A transdiagnostic comparison of trauma and panic memories in PTSD, panic disorder, and healthy controls. Journal of Behavior Therapy and Experimental Psychiatry, 40, $412-$ 
422.

Haidt, J., McCauley, C., \& Rozin, P. (1994). Individual differences in sensitivity to disgust: A scale sampling seven domains of disgust elicitors. Personality and Individual Differences, 16, 701-713.

Herrmann, N., \& Eryavec, G. (1994). Delayed onset post-traumatic stress disorder in World War II veterans. Canadian Journal of Psychiatry, 39, 439-441.

Lissek, S., Powers, A. S., McClure, E. B., Phelps, E. A., Woldehawariat, G., Grillon, C., \& Pine, D. S. (2005). Classical fear conditioning in the anxiety disorders: A meta-analysis. Behaviour Research and Therapy, 43, 1391-1424.

MacLeod, C., \& Mathews, A. (2004). Selective memory effects in anxiety disorders: An overview of research findings and their implications. In Reisberg D., Hertel P. (Eds.), Memory and emotion (pp. 155-185). New York: Oxford University Press.

Marks, I. M. (1969). Fears and phobias. Heinemann, London.

Miller, G. A. (1956). The magical number seven, plus or minus two: Some limits on our capacity to process information. Psychological Review, 6, 81-97.

Morgan, J. (2010). Autobiographical memory bias in social anxiety. Clinical Psychology Review, 30, 288-297.

Muris, P., \& Merckelbach, H. (1996). A comparison of two spider fear questionnaires. Journal of Behavior Therapy and Experimental Psychiatry, 27, 241-244.

Olatunji, B. O., Cisler, J., \& Phillips, M. L. (2010). Is disgust associated with psychopathology? Emerging research in the anxiety disorders. Psychiatry Research, 175, 1-10.

Olatunji, B. O., Huijding, J., de Jong, P. J., \& Smits, J. A. J. (2011). The relative contributions of fear and disgust reductions to improvements in spider phobia following exposure-based 
treatment. Journal of Behavior Therapy and Experimental Psychiatry, 42, 117-121.

Rachman, S. (1977). The conditioning theory of fear-acquisition: A critical examination. Behaviour Research and Therapy, 15, 375-387.

Radomksy, A.S., Ouimet, A.J., Ashbaugh, A.R., Lavoie, S.L., Parrish, C.L., \& O’Connor, K.P. (2006). Psychometric properties of the French and English versions of the Vancouver Obsessional Compulsive Inventory and the Symmetry Ordering and Arranging Questionnaire. Cognitive Behaviour Therapy, 35, 164-173.

Radomsky, A. S., \& Rachman, S. (1999). Memory bias in obsessive-compulsive disorder (OCD). Behaviour research and Therapy, 37, 605-618.

Radomsky, A. S., \& Rachman, S. (2004). The importance of importance in OCD memory Research. Journal of Behavior Therapy and Experimental Psychiatry, 35, 137-151.

Radomsky, A. S., Rachman, S., \& Hammond, D. (2001). Memory bias, confidence and responsibility in compulsive checking. Behaviour Research and Therapy, 39, 813-822.

Robinaugh, D. J., \& McNally, R. J. (2010). Autobiographical memory for shame or guilt provoking events: Association with psychological symptoms. Behaviour Research and Therapy, 48, 646-652.

Solomon, Z., Kotler, M., Shalev, A., \& Lin, R. (1989). Delayed onset PTSD among Israeli veterans of the 1982 Lebanon war. Psychiatry, 52, 428-436.

Szymanski, J., \& O’Donohue, W. (1995). Fear of Spiders Questionnaire. Journal of Behavior Therapy and Experimental Psychiatry, 26, 31-34.

Tabachnick, B. G., \& Fidell, L. S. (2007). Using Multivariate Statistics (5 $5^{\text {th }}$ ed.). Pearson Education Inc.: Boston, MA. 
Thordarson, D.S., Radomsky, A.S., Rachman, S., Shafran, R., Sawchuk, C.N., \& Hakstian, A.R. (2004). The Vancouver Obsessional Compulsive Inventory (VOCI). Behaviour Research and Therapy, 42, 1289-1314.

Wolpe, J. (1958). Psychotherapy by reciprocal inhibition. Stanford, CA: Stanford University Press. 
Table 1

Mean Scores by Condition on FSQ, SBQ, VOCI, BAI, BDI, and DS

\begin{tabular}{|c|c|c|c|c|c|c|}
\hline \multicolumn{7}{|c|}{ Condition } \\
\hline \multirow[b]{2}{*}{ Questionnaire } & \multicolumn{2}{|c|}{$\begin{array}{l}\text { Threat } \\
n=33\end{array}$} & \multicolumn{2}{|c|}{$\begin{array}{c}\text { No-Threat } \\
n=48\end{array}$} & \multicolumn{2}{|c|}{$\begin{array}{c}\text { Total } \\
n=81\end{array}$} \\
\hline & Mean & $S D$ & Mean & SD & Mean & SD \\
\hline FSQ & 26.48 & 31.34 & 30.40 & 33.26 & 28.80 & 32.35 \\
\hline SBQ & 1168.91 & 1230.32 & 1104.35 & 1198.47 & 1130.65 & 1204.30 \\
\hline SBQ-B $^{+}$ & 835.06 & 754.54 & 856.77 & 790.87 & 847.93 & 771.57 \\
\hline SBQ-T ${ }^{++}$ & 333.85 & 500.70 & 247.58 & 467.13 & 282.73 & 479.90 \\
\hline VOCI & 28.91 & 22.64 & 29.50 & 20.93 & 29.26 & 21.51 \\
\hline VOCI-CTN $^{+++}$ & 4.91 & 5.43 & 5.13 & 4.33 & 5.04 & 4.78 \\
\hline BAI & 9.03 & 7.35 & 9.15 & 7.06 & 9.10 & 7.14 \\
\hline BDI & 8.64 & 8.23 & 8.67 & 7.17 & 8.65 & 7.57 \\
\hline DS & $16.11 *$ & 4.10 & $18.21 *$ & 4.41 & 17.35 & 4.38 \\
\hline
\end{tabular}

Note. ${ }^{+}$Denotes the subscale of the SBQ that measures spider-related beliefs, ${ }^{++}$denotes the subscale of the SBQ that measures spider-related thoughts, ${ }^{+++}$denotes the subscale of the VOCI that measures contamination-related symptomatology; * indicates a significant difference $(p$ $<.05)$. 
Table 2

Mean Recall Memory Scores and Proportions by Condition, Time and Box Type

\begin{tabular}{ccccc}
\hline \multirow{2}{*}{ Box Type } & Threat & No-Threat & Threat & No-Threat \\
\hline Manipulated & $9.15(1.58)$ & $9.54(2.17)$ & $10.15(1.64)^{\mathrm{a}}$ & $10.27(2.17)$ \\
Un-manipulated & $8.70(1.90)$ & $9.44(1.83)$ & $9.24(2.01)^{\mathrm{a}}$ & $10.42(1.67)$ \\
Both & $17.85(2.46)$ & $18.98(3.00)$ & $19.39(2.73)$ & $20.69(2.96)$ \\
\hline $\begin{array}{c}\text { Proportionate } \\
\text { Memory }^{+}\end{array}$ & $0.51(0.07)$ & $0.50(0.07)$ & $0.53(0.07)^{\mathrm{b}}$ & $0.49(0.06)^{\mathrm{b}}$ \\
\end{tabular}

Note. ${ }^{+}$Adjusted means with covariates; ${ }^{\text {a,b }}$ corresponding letters indicate a significant difference $(p<.05)$. 


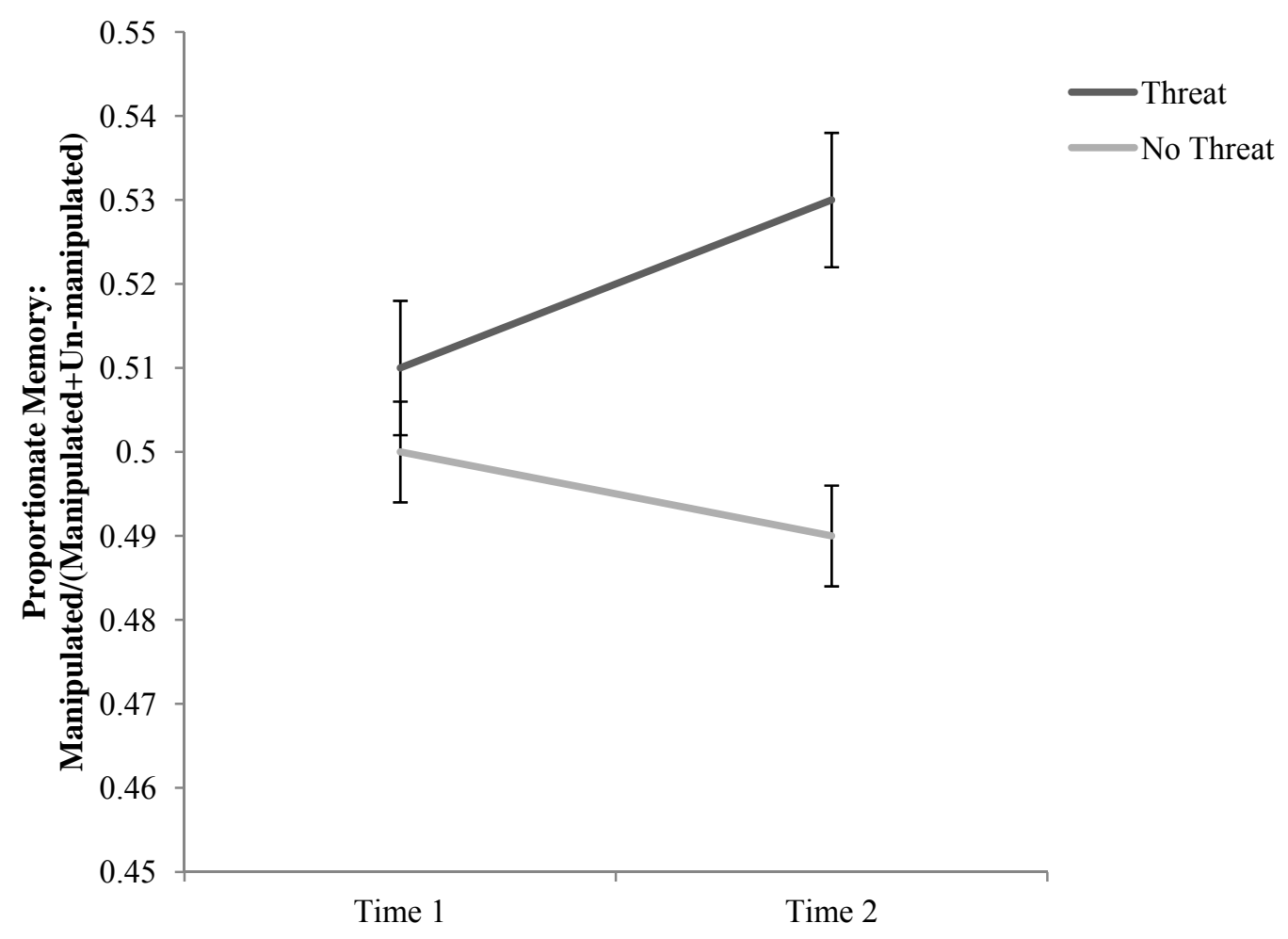

Figure 1. Proportion of manipulated to total items remembered by time and condition. 
Appendix
1) Clothes pin
2) Frog
3) Glass jar
4) Tennis ball
5) Toothpaste
6) Eraser
7) Bird
8) Light bulb
9) Hippo
10) $\mathrm{Car}$
11) Cactus
12) Tape
13) Nightlight
14) Padlock/Lock
15) Gum
16) Battery
17) Flower
18) Highlighter
19) Binoculars
20) Sunglasses
21) Comb
22) Mirror
23) Yo-yo
24) Pen
25) Bucket/Pail
26) Apple
27) Watermelon
28) Fish
29) Pineapple
30) Train 